\title{
Reply to "Comment on "A Vertical Exposure of the 1999 Surface Rupture of the Chelungpu Fault at Wufeng, Western Taiwan: Structural and Paleoseismic Implications for an Active Thrust Fault,' by Jian-Cheng Lee, Yue-Gau Chen, Kerry Sieh, Karl Mueller, Wen-Shan Chen, Hao-Tsu Chu, Yu-Chang Chan, Charles Rubin, and Robert Yeats," by Yuan-Hsi Lee, Shih-Ting Lu, Tung-Sheng Shih, and Wei-Yu Wu
}

by Jian-Cheng Lee, Charles Rubin, Karl Mueller, Yu-Chang Chan, Hao-Tsu Chu, Yue-Gau Chen, Kerry Sieh, Wen-Shan Chen, and Robert Yeats

Introduction

We welcome Y. H. Lee et al.'s interest in our article (Lee et al., 2001). We thank them for their comment, which provides a further opportunity for discussing the quantification of the slip amounts including horizontal and vertical components and the fault geometry for an earthquake thrust scarp in Wufeng, western Taiwan, during the 1999 M 7.6 earthquake.

In their comment, Y. H. Lee et al. used restoration of deformed concrete fence across the 1999 scarp to estimate the slip vector of the main fault. The estimated slip amount, especially the horizontal component, is different (significantly less) from our results presented in the 2001 BSSA article. They then applied an "area-balance" technique to compare their results with ours. They showed that their areabalance method favored their estimates including the slip amounts and the fault dip angle. They concluded that their estimated slip amounts are more reasonable than ours.

The fundamental questions in this issue, in our opinions, include the actual amounts of deformation (slip) and the associated deformation processes, as well as the limitation and uncertainty of the applied techniques on an earthquakeformed thrust scarp. Hereafter we attempt to answer these questions and clarify the related problems.

\section{Uncertainty of the Estimates}

First, we shall discuss the techniques of the estimates of the horizontal shortening used for Y. H. Lee et al. and for our previous article. It is important to know the limitations, the uncertainties, and the possible sources of errors for any estimate or calculation of the deformation, which enables us to evaluate the results. For our line-balancing method in the previous article, the uncertainties come mainly from the complicated deformation near the main fault zone, for in- stance, the overlapped structures and the ductile deformation. In particular, stretching and thinning of the sedimentary layers can be clearly observed around the core of the popup fold. The stretching effect of the depositional layers yields an overestimation of the actual amount of shortening across the thrust scarp. On the other hand, the missing and overlapped structures yield an underestimation of shortening amounts. The clearly observed stretching layers around the core of the small pop-up fold immediately east of the main scarp suggest a slight overestimation of horizontal shortening from our line-length measurement. Thus we acknowledge that we have seemingly yielded an overestimated amount of the horizontal shortening, $3.3 \mathrm{~m}$, which should be slightly less. However it is not likely to be half of this amount, as suggested by Y. H. Lee et al. This argument is also based on the observation of the deformation degree of the sedimentary layers involved in the estimates.

On the other hand, we are not able to evaluate the uncertainty of the techniques for the reconstruction of Y. H. Lee et al. Their Figure 1C illustrated the principle and the general idea of the reconstruction from the broken concrete fence. However, they did not describe in detail their measurements and calculation techniques. Without this information, it is difficult to evaluate their results. Even though the technique would be straightforward, there are always sources of error during the processes of making estimates. We cautiously anticipate that mistakes could occur due to the incompleteness of the broken fences. We speculate that their resulting horizontal shortening of $1.77 \mathrm{~m}$ might be too small. We will come back to this later.

We also want to discuss the area-balance method used by Y. H. Lee $e t a l$. They introduced the area-balance method to check the slip amounts yielded from our line-balance method and from their restoration of concrete fence. Their 

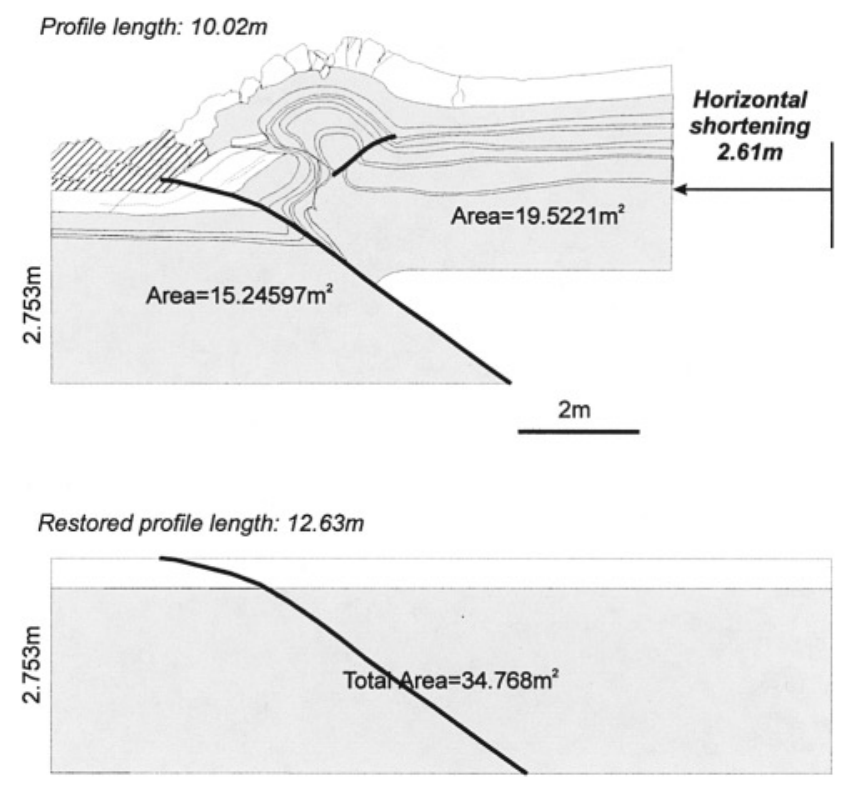

Figure 1. Restoration of the scarp and pop-up anticline based on area-balancing technique. The units of the complete assemblage (except the uppermost spoiled human soil) were used for retro-deformation. Based on the average thickness of $2.7 \pm$ $0.1 \mathrm{~m}$ and the area of $34.77 \mathrm{~m}^{2}$, it yields a estimate profile length of $12.6 \pm 0.2 \mathrm{~m}$ or $2.6 \pm 0.2 \mathrm{~m}$ of horizontal shortening.

area-balance method itself, in effect, cannot yield an estimate of the slip amounts but provides a tool to test the accuracy of the estimates of slip amounts and fault geometry. Y. H. Lee et al. argued that their results of $1.77 \mathrm{~m}$ horizontal shortening and $50^{\circ}$ dip angle gave the better fit for the areabalance check. However, there is a tradeoff between the dip angle and horizontal shortening in this area-balance technique. The same best fit can also be yielded by larger horizontal shortenings with lesser dip angles. Although the $3.3 \mathrm{~m}$ of horizontal shortening in our previous BSSA article seems to be overestimated, their $1.77 \mathrm{~m}$ horizontal shortening cannot be verified solely by their area-balance check-tool. Furthermore, their resulted fault dip angle of $50^{\circ}$ is much larger than the observed fault dip of $34^{\circ}-39^{\circ}$ in the excavation near the base of the trench.

\section{Another Area-Balancing Estimate}

We thus provide another area-balance technique, which is capable of yielding the amount of the horizontal shortening. The principle of this technique and its estimate for this particular Wufeng excavation are illustrated in Figure 1. The essence of this area-balancing technique is to use the same area, which also corresponds to the original horizontally lying sedimentary layers before and after the earthquake. This technique has also been broadly used in the pro- cess of restoration for balanced cross section (Woodward et al., 1989). Geometrically, on the vertical exposure in this case, the area for specific layers can be obtained by multiplying the length and the thickness. The amount of the area for the specific layers, which has deformed during the earthquake, would remain the same prior to the earthquake, assuming there was no significant density change. The amount of the area after the earthquake can be obtained by detailed mapping on the vertical exposure. We can then calculate the original length before the earthquake by dividing the amount of the area into the thickness of the specific layers. By comparing this with the present length, we obtain the horizontal shortening parallel to the exposure for these layers. Because details of this technique are presented in a separate article (Lee et al., 2003), we show only the results from this areabalance method (Fig. 1). This method yielded a horizontal shortening of $2.6 \pm 0.2 \mathrm{~m}$, which is between the estimates from Y. H. Lee et al. and from our previous line-length method.

Considering the geometry of the involved deformation structures across the thrust scarp, including the major fault, secondary faults, and fold, we can further differentiate the deformation processes into two stages. The lower level dealt with the slippage on the $39^{\circ}$ east-dipping main thrust fault with 2.1-2.3 m of horizontal shortening and $1.8 \mathrm{~m}$ of vertical displacement. The upper level, consisting of a high-angle wedge thrust, two opposing secondary thrusts, and an associated pop-up anticlinal fold, provides an additional deformation with $0.3-0.5 \mathrm{~m}$ of horizontal and vertical components of movement. Because the results of our later area-balance method are rather consistent with the geometry and the kinematics of the deformation structures observed in the excavation, we are more comfortable with these results.

\section{Comparison with the Neighbor Site}

Y. H. Lee et al. also made an estimate of surface slip motion by reconstruction in a neighboring parking lot, some $250 \mathrm{~m}$ south of the excavation site. They obtained a horizontal slip of $2.5-2.67 \mathrm{~m}$ and a vertical displacement of $1.3-1.5 \mathrm{~m}$, which yields an averaged fault dip angle of $28^{\circ}$. At the same location, we obtained similar (though slightly larger) amounts of horizontal slip $(2.82 \pm 0.40 \mathrm{~m})$ and vertical offset $(1.62 \pm 0.06 \mathrm{~m})$ (Angelier et al., 2003), which yielded a fault dip angle of $30^{\circ}$. The amounts of the horizontal shortening from both reconstructions in the parking lot are close to our latest estimate from the area-balance technique for the excavation site (see comparison in Table 1).

Although it is not necessary that the surface fault motions be identical on the two neighboring sites (for instance, there was stronger localized anticlinal folding near the major scarp at the excavation site), the results of reconstruction of the slip amounts in the parking lot site suggest that our latest 
Table 1

Estimates of Slip Amounts and Dip Angle of the Primary Thrust Fault During the 1999 Chi-Chi Earthquake in the Excavation Site and the Parking Lot Site, Wufeng City, Western Taiwan*

\begin{tabular}{lccccccc}
\hline & \multicolumn{3}{c}{ Excavation site } & & \multicolumn{2}{c}{ Parking lot site } \\
\cline { 2 - 3 } \cline { 6 - 7 } & Lee et al., 2001 & Y. H. Lee et al. & Our revised model & & Y. H. Lee et al. & Angelier et al., 2002 \\
\hline Horizontal shortening $(\mathrm{m})$ & $3.3 \pm 0.3$ & 1.77 & $2.6 \pm 0.2$ & & $2.5-2.67$ & $2.82 \pm 0.40$ \\
Vertical offset (m) & $2.2 \pm 0.1$ & 2.1 & $2.2 \pm 0.1$ & & $1.3-1.5$ & $1.62 \pm 0.06$ \\
Total slip (m) & $4.0 \pm 0.2$ & 2.75 & $3.4 \pm 0.2$ & & $2.8-3.06$ & $3.25 \pm 0.38$ \\
Thrust dip-angle ( $\left.{ }^{\circ}\right)$ & $34 \pm 3$ & 50 & $39 \pm 2$ & & 28 & 30 \\
\hline
\end{tabular}

*Three estimates are presented for the excavation site, including from our previous article, Y. H. Lee et al.'s comment, and our revised model of area-balance technique. Two estimates are presented for the parking lot site, $250 \mathrm{~m}$ south of the excavation site along the strike of fault.

area-balance estimate provides the best agreement in terms of the geometry of the basal thrust and the slip amounts. This provides another favorable factor that we prefer to the estimates determined by our area-balance method.

\section{Further Discussion}

Assuming Y. H. Lee et al.'s calculation from the broken fence on top of the surface scarp was correct, there are still other possibilities for explaining the discrepancy between the different techniques of estimates. One likely possibility is that the discrepancy was due to strain transfer, from the semiductile deformation in the soil and sand materials of the alluvium deposits to the brittle ruptures of the concrete fence on top of the surface. In this case, the deposits of the soil and sand layers have absorbed more deformation than the concrete fence. This brings up the issue of decoupling along the boundary of different deformed materials and their response to deformation with various rheology during earthquake faulting. These phenomena have also been described at several places along the 1999 surface ruptures (Kelson et al., 2001). It would be interesting to discuss the difference between the deformation on the surface level and that on the subsurface level; however, it is beyond the scope of the present study.

Finally, there is a possibility that Y. H. Lee et al. have made some mistakes on the restoration of the broken fence. This speculation also came from the fact that we found a segment of missing fence buried completely within the soil deposits in the core of the deformation zone beneath the scarp. If they indeed missed this $\sim 0.7$-m-long segment of fence during the measurement, their result of the horizontal slip would be $2.47 \mathrm{~m}$, rather close to our latest estimate. However, this speculation cannot be confirmed without details of their measurements.

\section{Acknowledgments}

Financial support for our work on the 1999 Chi-Chi earthquake surface ruptures was provided by National Science Council Grants NSC882116-M001-037 and NSC89-2116-M001-037. This is a contribution of the Institute of Earth Sciences, Academia Sinica, IESAS840.

\section{References}

Angelier, J., J. C. Lee, H. T. Chu, and J. C. Hu (2003). Reconstruction of fault slip of the September 21st, 1999, Taiwan earthquake in the asphalted surface of a car park, and co-seismic slip partitioning, J. Struct. Geol. 25, no. 3, 345-350.

Keith I. Kelson, K.-H. Kang, W. D. Page, C.-T. Lee, and L. S. Cluff (2001). Representative styles of deformation along the Chelungpu Fault from the 1999 Chi-Chi (Taiwan) Earthquake: geomorphic characteristics and responses of man-made structures. Bull. Seism. Soc. Am. 91, no. 5, 930-952.

Lee, J. C., Y. G. Chen, K. Sieh, H. T. Chu, K. Mueller, Y. C. Chan, C. Rubin, R. Yeats, and W. S. Chen (2001). A vertical exposure of the 1999 surface rupture of the Chelungpu Fault at Wufeng, Western Taiwan: structural and paleoseismic implications for an active thrust fault. Bull. Seism. Soc. Am. 91, no. 5, 914-929.

Lee, J. C., C. Rubin, K. Mueller, Y. G. Chen, H. T. Chu, Y. C. Chan, and K. Sieh (2003). Quantitative analysis of the movement along an earthquake thrust scarp: a case study on a vertical exposure of the 1999 surface rupture of the Chelungpu Fault at Wufeng, Western Taiwan. J. Asian Earth Sci. (in review).

Woodward, N., S. Boyer, and J. Suppe (1989). Balanced geological crosssections: an essential technique in geological research and exploration, in 28th International Geological Congress, Washington, D.C., $132 \mathrm{pp}$.

Institute of Earth Sciences

Academia Sinica

P.O. Box 1-55

Nankang, Taipei, R.O.C., Taiwan

(J.-C.L., Y.-C.C.)

Department of Geology

Central Washington University

Ellensberg, Washington

(C.R.) 
Department of Geology

University of Colorado

Boulder, Colorado

(K.M.)

Central Geological Survey

P.O. Box 968

Taipei, R.O.C., Taiwan (H.-T.C.)

Department of Geology

National Taiwan University

Taipei, R.O.C., Taiwan

(Y.-G.C., W.-S.C.)
Division of Geological and Planetary Sciences

100-23

Caltech

Pasadena, California

(K.S.)

Department of Geosciences

Orgon State University

Corvallis, Oregon

(R.Y.) 